\title{
Association of factors on parental knowledge and practice regarding Anthelmintic drugs in a Tertiary Hospital, Sri Lanka
}

\author{
Thanujah Murugathas ${ }^{1}$, Nivethika Baskaran ${ }^{1}$, P.A.Dinesh Coonghe ${ }^{2}$, Manoji Gitanjali \\ Sathiadas $^{2}$, Sinnadurai Thuvaragan ${ }^{1}$ \\ ${ }^{1}$ Department of Pharmacy, Faculty of Allied Health Sciences, University of Jaffna, Sri Lanka, \\ thanujahmurugathas03@gmail.com,nivethiha95@gmail.com,sthuvaragan@univ.jfn.ac.lk
}

${ }^{2}$ Faculty of Medicine, University of Jaffna, Sri Lanka, padcoonghe@univ.jfn.ac.lk,docsathiadas@hotmail.com 


\title{
ASSOCIATION OF FACTORS ON PARENTAL KNOWLEDGE AND PRACTICE REGARDING ANTHELMINTIC DRUGS IN A TERTIARY HOSPITAL, SRI LANKA
}

\begin{abstract}
Worm infestations are one of the significant health problems affecting children in developing countries. It is highly prevalent among poverty-stricken communities due to poor hygiene. The objective of this study was to assess the knowledge and practice on anthelmintic drugs and the influence of selected factors and knowledge on worm infestation among parents of pediatric patients attending OPD at a Teaching Hospital, Sri Lanka. It was a descriptive cross-sectional study. A validated interviewer-administered questionnaire was used as a study instrument. A systematic random sampling method was used to select the 422 participants. The response rate was $79 \%$ $(\mathrm{n}=334)$. Pre-determined cut-off values used to assess the knowledge of worm infestation and knowledge on anthelmintic drug usage were $60 \%$ and $50 \%$, respectively. Using these cut-off values, the level of knowledge was categorized as good and poor. Most of the participants were females $(85.6 \%, n=286)$, and the mean age was $32( \pm 5)$ years. $60.2 \%(n=201)$ and $86.8 \%(n=290)$ had good knowledge on worm infestation and anthelmintic drug usage. Only $66.4 \%(n=215)$ of respondents used anthelmintics to their children as self-medication or prescribed drug. The age of the participants was statistically associated with anthelmintic drug knowledge $(p<0.05)$. Knowledge of worm infestation could be further improved by educating the parents.
\end{abstract}

Keywords: Anthelmintic drugs, Worm infestation, Knowledge, Practice

\section{Introduction}

Intestinal worm infestations are a common global problem in the world. Intestinal worms are widely prevalent in tropical and subtropical countries. It mainly affects the most deprived communities having poor sanitation. Morbidity is directly linked with worm burden, where greater the number of worms, the greater the severity of the disease. Intestinal worm infestation is caused by eating undercooked meat from an infected animal, consuming contaminated water, consuming soil contaminated food, contacting contaminated feces, poor sanitation, and poor hygiene. The common symptoms associated with intestinal worms are abdominal pain, diarrhea, nausea, vomiting, weakness, and unexplained weight loss (WHO, 2020 ). The common intestinal Helminthes in the world are roundworm (Ascaris lumbricoid), whipworm (Trichuris trichiura), and hookworms (Necator americanus and Ancylostoma duodenale). According to the worldwide estimation in 2010, approximately 820 million persons were infected with $A$. lumbricoides, 460 million with $T$. trichiura, and 440 million with $A$. duodenale or $N$. Americanus. As a developing country with a warm, wet, tropical climate Sri Lanka is endemic for all three intestinal worm types, excluding A. duodenale (De Silva, 2018).

Children are unshielded for certain diseases, and it increases morbidity and mortality rates. These diseases comprise diarrhea, vector-borne diseases, helminthic infection, respiratory infections, and injuries (Prathaban, 2010). Worm infestation mainly occurs due to a deficiency in sanitary facilities, 
hazardous human waste disposal systems, the inadequacy of safe water supply, and low socioeconomic status. Intestinal worms can contaminate through ingestion of parasite eggs or skin contact with motile larva. The groups at risk are pre-school children and school-aged children. These infections produce adverse effects on health, growth, and learning ability with a reduction in physical fitness and impaired memory and cognition. (Nath, Padmawati and Shafiul, 2018)

The universal strategy for addressing the intestinal worm infestation is mass deworming with anthelmintic drugs periodically administered to school-aged children and other high-risk groups (Andrews, Bogoch, and Utzinger, 2016). Deworming is not the only solution; however, upgrading basic hygiene, sanitation, health education, and providing access to safe drinking water are also keys to settle the nutritional problems raised by intestinal worms (WHO, Department of Nutrition for Health and Development).

Anthelmintic drugs are indicated in the treatment of worm infestation. The drug recommended for intermittent deworming includes Levamisole, Mebendazole, Albendazole, and Pyrantel Pamoate. However, Benzimidazoles such as Albendazole and Mebendazole are the most commonly used anthelmintics. Albendazole and Mebendazole have a very similar mode of action and very similar efficacy after a single administration (Keiser and Utzinger, 2008).

Deworming aims to reduce initial worm load by less than $80 \%$, eliminate the disease, and repeat treatment is essential to prevent heavy loads from being re-accumulated (Hall and Horton, 2009). According to the guidelines for deworming children and pregnant women in the community setting during 2013-2016, the country was divided into high-risk areas (Uva, Sabaragamuva, and central provinces) and moderate risk (all other provinces). The guidelines recommend that all children in high-risk areas be treated twice a year with a single dose of Mebendazole (500mg), while children in moderate risk areas should be treated once a year (WHO, 2004).

The people of Sri Lanka have inaccurate beliefs about the transmission and treatment of worm infestations. It leads to the over usage of anthelmintic drugs. (Perera et al., 2012). In Sri Lanka, only a few studies were conducted on the knowledge and practice regarding worm infestation and deworming. This study aims to assess the knowledge and practice regarding anthelmintic drugs among the parents of pediatric patients admitted to Teaching Hospital, Jaffna, and identify the influence of social demographic factors and knowledge on worm infestations on them. Assessing their knowledge and practice on Anthelmintic drugs is beneficial in averting worm infestation among children.

\section{Methodology}

\section{Study Design}

It was a hospital-based descriptive cross-sectional study among the parents attending Outpatient Department (OPD) and OPD pharmacy, pediatric counter of Jaffna's Teaching Hospital. Data were collected via an interviewer-administered questionnaire that lasted 15 minutes. 


\section{Study setting and sampling}

According to the study conducted in Sri Lanka (Perera et al., 2012), out of 200 mothers, 55.3\% gave anthelmintic treatment every 6 months. Thus, the proportion value was taken as 0.553 . The sample size was calculated from According to the study conducted in Sri Lanka (Perera et al., 2012), out of 200 mothers, $55.3 \%$ gave anthelmintic treatment the formula: $n=\frac{Z^{2} p(1-p)}{d^{2}}$ where, $\mathrm{n}=$ sample size, $\mathrm{Z}=$ criteria value of specific confidence $(95 \%), \mathrm{p}=$ preliminary estimation of proportion of a given characteristics (0.553) and $\mathrm{d}=$ Acceptable amount of absolute error (0.05). The sample size was 380, and $10 \%$ non-responders' rate was expected. Finally, the sample size was 422 .

The parents of pediatric patients attending the OPD at the Teaching hospital of Jaffna during the study period were recruited. The average number of pediatric patients under the age of 12 years attending the Out-Patients Department per month was 4500. The first patient's parent was selected randomly from the first ten parents of pediatric patients and continued with every 10th patient's parent via systematic sampling technique. Interviewer administered questionnaire was administered to every selected parent of the pediatric patient, and privacy was ensured.

\section{Study instrument}

An interviewer-administered questionnaire was used to collect data. The questionnaire was developed in English and validated by three experts. It was translated into Tamil and Sinhala by a language expert. The questionnaire consisted of three sections, namely A, B, C, and D. Section A was designed to collect data on the participants' socio-demographic and economic factors. It consisted of twelve questions. Section B consisted of questions regarding the knowledge of worm infestation. Section C and Section D consisted of questions about knowledge and practice on anthelmintic drug usage, respectively.

\section{Data collection}

Data collection was carried out among the parents after obtaining the ethical clearance from the Ethical Review Committee, Faculty of Medicine, University of Jaffna, and permission from the Director, Teaching Hospital Jaffna. Data collection was done among the parents of pediatric patients attending OPD at Teaching Hospital Jaffna.

\section{Data analysis}

The collected data were entered into SPSS 23 (Statistical Package for Social Sciences version 23). The research problems, specific objectives, and variables were analyzed. To assess the knowledge, a score was provided for each question of sections B and C. Each section B question shows that knowledge on worm infestation carried five marks for correct response and zero marks for correct or 'do not know' responses. A total of thirty-five marks was provided for this section. The knowledge level was categorized as "Good knowledge" and "Poor knowledge" with a cut-off value of $60 \%$ or above and below $60 \%$, respectively. Section $\mathrm{C}$ knowledge on anthelmintic drug consisted of six questions, carrying 100 scores was categorized with a cut-off value of $50 \%$ as good and poor 
knowledge. Findings of practice, section D was expressed in frequencies and percentages. The influence of associated factors on knowledge was determined by the Chi-square test, and $95 \%$ of the confidence interval was set for the test. With this, the result was significant if $p$-value $<0.05$.

\section{Ethical considerations}

The research proposal was submitted to Ethical Review Committee through, Head, Department of Pharmacy, Dean, Faculty of Allied Health Sciences, University of Jaffna, and the approval was obtained to carry out this study. Permission for the data collection of this study was obtained from the Director, Teaching hospital, Jaffna. The purpose of the study was explained to the participants, and written consent was received.

\section{Results}

The response rate of this study was $79 \%$. The majority of the participants were Females $(85.6 \%)$ with a mean age of $32( \pm 5)$ years. Sri Lankan Tamils $(98.8 \%)$ following Hinduism $(82.9 \%)$ were prominent among the Participants. More than half of the participants were housewives (58.1\%) where a large proportion of the participant's spouses worked (89.2\%). Educational qualifications of more than half of the respondents (53.3\%) and their spouses $(60.7 \%)$ were up to G.C.E A/L. Approximately half of the participant's (49.7\%) salary was between 30000 to 50000 LKR. More than two-thirds of the participants were having a nuclear family $(68.3 \%)$, and a nearly equal portion of the participants had one child (50.9\%) and more than one child (49.1\%)

Table 1: Distribution of socio-demographic factors of the study population ( $n=334)$

\begin{tabular}{lcc}
\hline Factors & Frequency & Percentage (\%) \\
\hline Relationship of the respondent to child & & \\
$\quad$ Father & 48 & 14.4 \\
$\quad$ Mother & 286 & 85.6 \\
Age & & \\
$\quad$ Below 30 years & 143 & 42.8 \\
$\quad 30$ and Above & 191 & 57.2 \\
Ethnicity & & \\
$\quad$ Sri Lankan Tamil & 330 & 98.8 \\
$\quad$ Sri Lankan Muslim & 4 & 1.2 \\
Religion & & 82.9 \\
$\quad$ Hinduism & 277 & 15.9 \\
$\quad$ Catholicism & 53 & 1.2 \\
$\quad$ Islam & 4 & 41.9 \\
$\quad$ Wccupation of Respondent & & 58.1 \\
$\quad$ Horkers & 140 & \\
\hline
\end{tabular}




\begin{tabular}{lcc}
\hline Occupation of Spouse & & \\
$\quad$ Workers & 298 & 89.2 \\
$\quad$ Homemakers & 36 & 10.8 \\
Educational Qualification of Respondent & & 53.3 \\
$\quad$ Up to G.C.E A/L & 178 & 46.7 \\
$\quad$ G.C.E A/L and Degree & 156 & 60.7 \\
Educational Qualification of Spouse & 203 & 39.3 \\
$\quad$ Up to G.C.E A/L & 131 & 41.3 \\
$\quad$ G.C.E A/L and Degree & 138 & 49.7 \\
Family Monthly Income & 166 & 9.0 \\
$\quad<30,000$ & 30 & \\
$\quad 30,000-50,000$ & & 68.3 \\
$\quad>50,000$ & 228 & 31.7 \\
Type of family & 106 & 50.9 \\
$\quad$ Nuclear & & 49.1 \\
$\quad$ Extended & 170 & \\
Number of children under 12 years & 164 & \\
$\quad$ One &
\end{tabular}

\section{Knowledge of worm infestation}

More than half of the participants (62.6\%) knew what the worm infestation was, and only one-fourth listed the names of worms $(n=82,24.6 \%)$ while $74.9 \%$ believed that earthworm causes harm to the children. $56.6 \%$ (189) knew that worms live inside the intestines. More than three-fourths $(76.6 \%)$ mentioned that poor cleanliness is the cause of worm infestation. Also, they listed the modes of transmission of worm infestation as follows, drinking contaminated water (98.2\%), Consuming contaminated food $(99.1 \%)$, Poor hand hygiene (100.0\%), walking barefooted $(98.2 \%)$, and contacting with soil $(100.0 \%)$. There were many signs and symptoms associated with worm infection, and an average of $79.28 \%$ was well informed regarding this. Itching around the anus $(87.4 \%)$, abdominal pain $(71.0 \%)$, loss of appetite $(79.0 \%)$, body weakness $(71.6 \%)$, and sleeping disturbance (87.4\%). Everyone was conscious of the method of preventing the occurrence of worm infestation. On the whole, $60.2 \%(n=201)$ had good knowledge of worm infestation.

Table 2: Distribution of knowledge of the participants regarding worm infestation $(n=334)$

\begin{tabular}{lcc}
\hline Category & N & Percentage (\%) \\
\hline What is a worm infestation & 209 & 62.6 \\
Identifying one or more worm name & 82 & 24.6 \\
An earthworm is harmless to children & 84 & 25.1 \\
\hline
\end{tabular}




\begin{tabular}{lcc}
\hline Place where worms live in the human body & 189 & 56.6 \\
Cause of worm infestation & 256 & 76.6 \\
Modes of transmission and route of entry of worm & 328 & 79.3 \\
Signs and symptoms of worm infestation & 265 & 100.0 \\
How can you prevent the worm infestation & 334 & \\
\hline
\end{tabular}

\section{Knowledge of the usage of anthelmintic drug}

Table 3: Distribution of knowledge of the participations regarding usage of the anthelmintic drug $(n=334)$

\begin{tabular}{lcc}
\hline Category & N & Percentage (\%) \\
\hline Name of the drug & 308 & 92.2 \\
Frequency of taking the drug & 287 & 85.9 \\
Available dosage forms & 206 & 61.7 \\
Taking the drug together with family & 303 & 90.7 \\
Repeating dose after 10 to 14 days & 227 & 68.0 \\
Not to be used for children under one and a half years & 300 & 89.8
\end{tabular}

A significant number of participants (92.2\%) named the drug they used, and $85.9 \%$ knew the interval of taking the drugs. Regarding the dosage forms, $61.7 \%$ correctly mentioned the different dosages, while $25.4 \%$ gave partially correct answers. A substantial number $(90.7 \%)$ of the participants knew that anthelminthic should be taken together by the whole family, and $68.0 \%$ were sure that the next dose should be repeated after 10 to 14 days. $89.8 \%$ of the participants were well versed that children under one and a half years should not be given anthelmintic drugs. Overall knowledge of participants on usage of anthelmintic drugs was good $(86.8 \% \mathrm{n}=210)$. The age of the participants was statistically associated with anthelmintic drug knowledge $(\mathrm{p}<0.05)$.

\section{Practice Regarding the usage of anthelmintic drugs}

Among the participants, 215 (64.4\%) respondents gave the anthelmintic drug to their children. The remaining reasoned that their children were below one and half years $106(31.7 \%)$ and 13(3.9\%) never deemed it necessary. The respondents administered the anthelmintic drugs in different time intervals like every three months 50(23.3\%), every six months 63(29.3\%), one year 13(6.0\%), and whenever necessarily $72(33.5 \%)$. They followed both self-medication $149(69.3 \%)$ and prescription $100(46.5 \%)$. Participants followed with self-medication knew the dosage of the drug by their previous 
experience. 211(98.1\%) participants took the drug with their whole family, and 181(84.2\%) repeated the doses after 10 to 14 days.

\section{Discussion}

This study focused on the knowledge and practice on the usage of anthelmintic drugs and the influence of selected socio-demographic factors and knowledge on worm infestation among parents of pediatric patients attending OPD at Teaching Hospital, Jaffna. The study was done among 334 parents. The mean age of participants was $32 \pm 5$ years with an age range of 21 to 44 years. More than half of the participants were above 30 years $(57.2 \%)$, and most $(85.6 \%)$ were mothers. It is in line with a study where most participants were mothers $(81.22 \%)$ with a mean age of $30.47(\mathrm{SD}=5.47)$ years. (Aniwada et al., 2016). In contrast to a study where $23.1 \%$ were above 30 years, $76.9 \%$ were below 30 years. (Amar et al., 2013). The majority of the participants (82.9\%) were Hindus and Christians (15.9\%). It is different from another study where $98.3 \%$ were Hindus and the remaining 1.7\% Christian. (Amar et al., 2013).

In the present study, $60.2 \%(n=201)$ of participants had good knowledge regarding worm infestation. In contrast, a community-based descriptive cross-sectional study conducted in selected slums of Pune city in India was manifested with average knowledge(75.0\%), poor knowledge (22.0\%), and good knowledge (3.0\%) regarding worm infestation. (Ray, Shine, and N, 2016). 62.6\% of the participants mentioned worm infestation, which is lower than other studies in which 364 (88.8\%) heard about worms, and 261(63.7\%) knew about deworming. (Aniwada et al., 2016). In another study, 131 respondents (81.9\%) heard about it. (Nath et al., 2018) Participants listed the different modes of transmission like drinking contaminated water (98.2\%), Consuming contaminated food $(99.1 \%)$, Poor hand hygiene (100.0\%), walking barefooted (98.2\%). Another study presents its finding, where $89(24.5 \%)$ of the parents knew that worms could be caused by walking barefooted (Aniwada et al., 2016)

It was found that participants knew that worm infestation caused itching around the anus and sleep disturbance (87.4\%), abdominal pain (71.0\%), loss of appetite $(79.0 \%)$, and body weakness $(71.6 \%)$. In another study, parents reported a similar thing, abdominal discomfort (67.9\%), and identified that the pupil infected with the worm eats without gaining weight (52.5\%)(Aniwada et al., 2016). It is incoherent to a study where participants reported pain abdomen (23.0\%), perianal itching (19.6\%), vomiting $(23.0 \%)$, and worms in the stool $(14.8 \%)$ as the signs and symptoms of worm infestations. (Amar et al., 2013). Another study suggested abdominal pain (31.5\%), delay in growth (6.5\%), itching in feet $(4.0 \%)$, chronic cough (1.5\%), abdominal distention (11.0\%), hypopigmented patches $(19.0 \%)$, sleepiness (13.0\%) and vomiting (15.0\%) (Perera et al., 2012).

In this study, $89.8 \%$ of parents believed that eating sugar can cause intestinal parasites in children's stomachs. 52.1\% of participants mentioned eating sugary foods as a significant cause of worm infestation. Also, 158(43.3\%) believing that it can be contracted through eating sweet foods. (Amar et al., 2013). However, it must be noted that it is an entirely misplaced belief. The fact is that intestinal parasites can invade the child's body regardless of the amount of sugar the child consumes. Many participants mentioned that consuming contaminated food $(99.1 \%)$ and poor hand hygiene $(100.0 \%)$ 
were the cause of worm infection. It was found low in another study where participants reported eating food contaminated with soil (1.7\%) and eating with unclean hands (10.3\%). (Amar et al., 2013). In contrast, mothers mentioned swallowing parasite eggs $(67.0 \%)$, piercing the skin $(6.0 \%)$, playing with sand $(51.0 \%)$, eating sweets $(38.5 \%)$, poor general hygiene $(18.0 \%)$, and eating raw scraped coconut (5.5\%) might cause worm infection. (Perera et al., 2012). In a study, $64.9 \%$ of mothers reported hand washing before eating and feedings, 58.1\% notified hand washing after defecation, $57.2 \%$ regular use of footwears by self and children, $83.0 \%$ reported maintaining food hygiene, and $40.2 \%$ practiced sanitary latrine to prevent worm infection of their children. (Amar et al., 2013). It was dissimilar to our study, where the total population had complete knowledge of preventive methods.

This present study revealed that most of the study participants (86.8\%) had good knowledge and others (13.2\%) had poor knowledge of anthelmintic drug usage. About (91.3\%) participants knew about the drug used to treat the worm infestation, and (86.3\%) of respondents knew about the drug's dosage. The study was conducted in residents of Jos, Plateau State, Nigeria, where (70.4\%) respondents knew about the drug and $(96.2 \%)$ of respondents knew about the dosage of the drug (Jimam et al., 2013). More than half of the respondents, 215 (64.4\%), gave the anthelmintic drug to their children, and $106(31.7 \%)$ respondents skipped giving the drug to their children as they were below one and half years of age. It was found to be high in a study; 168 (84.0\%) reported giving routine anthelmintic treatment to their children (Perera et al., 2012). Anthelmintic drugs were recommended for children above 18 months of age. (Deworming guideline, 2012).

Out of the 215 respondents, $23.3 \%$ used the drug every three months, while $29.3 \%$ used it after six months. A significant number of participants gave anthelmintic treatment every three months $(39.3 \%)$ and every six months (55.3\%) (Perera et al., 2012). According to prescription, nearly $69.3 \%$ of the respondents used the anthelmintic drug as self-medication. Most of them (98.1\%) took the anthelmintic drugs with all the family members, and $84.2 \%$ of respondents repeated the dose after 10 to 14 days. Similarly, a cross-sectional study was done in the suburban area of Sri Lanka, where (84.0\%) respondents reported giving routine anthelmintic treatment to their children. Only $30.0 \%$ had a repeat course after 10 to 14 days. Among them, $70.5 \%$ of respondents obtained the anthelmintic treatment from their general practitioner, and $80.5 \%$ received treatment without a prescription (Perera et al., 2012).

\section{Conclusion}

The study's findings state that $86.8 \%$ (290) had good knowledge of the usage of anthelmintic drugs and $60.2 \%(n=201)$ had good knowledge of worm infection. Around $64.4 \%$ of respondents administered anthelmintic drugs for their children, and among them, nearly $67.4 \%$ of respondents used it within one year. Of the children who received routine anthelmintic treatment, $98.1 \%$ took with the whole family, and $84.2 \%$ had a repeat anthelmintic course. Overall, knowledge was good on the usage of the anthelmintic drug. However, the level of knowledge of the remaining participants can be improved by frequent education and counseling. 
Thanujah Murugathas /Association of Factors on Parental Knowledge and Practice Regarding Anthelmintic Drugs in a Tertiary Hospital, Sri Lanka

\section{Acknowledgment}

The authors acknowledge the contribution of Consultants for validating the questionnaire, The Director, Teaching Hospital, Jaffna, participants, and those involved in various junctures throughout the research project.

\section{References}

Andrews, J.R., Bogoch, I.I. and Utzinger, J., 2017. The benefits of mass deworming on health outcomes: New evidence synthesis, the debate persists. The Lancet Global Health, 5(1), pp.e4-e5.

Aniwada, E.C., Uleanya, N.D., Igbokwe, L.N. and Onwasigwe, C., 2016. Soil-transmitted helminths; prevalence, perception, and determinants among primary school children in rural Enugu State, Nigeria. International Journal of Tropical Disease and Health, 15(1), pp.1-12.

Chinyem, M.U., Friday, U.A., Ibrahim, T.B., Chinedu, I.A., Ndubueze, A.B., Chukwuemeka, M.C., and Kasimu, K., 2017. Frequency of Deworming, Parental Perception, and Factors Associated with the Practice of Deworming School-age Children in North-East Nigeria. International Journal of Tropical Disease \& Health, 24(4), pp.1-10.

De Silva, N.R., 2018. The soil-transmitted helminths in Sri Lanka: A review Of the Recent Literature. Sri Lankan Journal of Infectious Diseases, 8(2).

Jimam, N.S., Wetkos, D.D., Falang, K.D., David, S. and Akpor, O.J., 2013. Assessment of the knowledge, attitude, and awareness of residents of Jos, Plateau State, Nigeria, towards worm infestation and deworming.

Keiser, J. And Utzinger, J., 2008. Efficacy Of current drugs against soil-transmitted helminth infections: Systematic review and Meta-Analysis. Jama, 299(16), pp.1937-1948.

Masaku, J., Mwende, F., Odhiambo, G., Musuva, R., Matey, E., Kihara, J.H., Thuita, I.G. and Njomo, D.W., 2017. Knowledge, practices, and perceptions of geo-helminthes infection among parents of pre-school age children of the coastal region, Kenya. PLoS neglected tropical diseases, 11(3), p.e0005514.

Nath, T.C., Padmawati, R.S., Alam, M.S., Das, S. and Murhandarwati, E.H., 2018. Elimination of soiltransmitted helminthiasis infection in Bangladesh: knowledge, attitudes, and practices regarding mass drug administration. Journal of Global Health Reports, 2.

Perera, P.J., Disanayake, D., Fernando, M.P., Warnakulasoority, T.D. and Ranathunga, N., 2012. Knowledge and practices related to helminth infections among mothers living in a suburban area of Sri Lanka. Southeast Asian Journal of Tropical Medicine and Public Health, 43(6), p.1326.

Prathaban, S., 2010. A study to assess the knowledge and practices regarding worm infestation among the mothers of school-age children (6-12 years) to develop a health education pamphlet at Paruvachi, Erode District (Doctoral dissertation, College of Nursing, Dharamarathnakara Dr. Mahalingam Institute of Paramedical Sciences and Research, Erode).

Tripura, A. and Reang, T., 2013. A study of knowledge and practice on intestinal helminthiasis among rural tribal mothers of under-five children in Mohanpur block, west district of Tripura: India's northeastern state. Journal of Evolution of Medical and Dental Sciences, 2(47), pp.9081-908.

WHO, Department of Nutrition for Health and Development (NHD), Date of access: 20/04/2020, https://www.who.int/nmh/about/nhd/en/

WHO, Deworming for Health and Development, Date of access: 20/04/2020, https://apps.who.int/iris/bitstream/handle/10665/69005/WHO_CDS_CPE_PVC_2005.14.pdf;jsessionid=DFF9E 7BA4DE9CF38DF1B67C4313BACA0? sequence $=1$

WHO, Soil-transmitted helminthiases, Date of access: 20/04/2020,

https://www.who.int/intestinal_worms/more/en/ 